Conference Article

\title{
Effect of Vibrations of Instrument Tools and a Treated Workpiece a on Wear Rate and Quality of a Machined Surface
}

\author{
S.I.Fecak and Yu.V.Idrisova
}

Ufa state aviation technical university, Ufa, Russia - 450008

Received 2 September 2015; Accepted 11 September 2015

\begin{abstract}
This article presents a technique to create a model of shaping of the machined surface that takes into account: tool wear, dynamic system of the metal-cutting machine, cutting parameters, manufacturing errors and assembly errors of the metalcutting machine's drives.

Keywords: spectrum of external perturbations, elastic system, cutting process, tool wear resistance, self-oscillations, forming, the dynamic characteristics of the machine, waviness and roughness of the workpiece surface.
\end{abstract}

\section{Introduction}

Productivity and the required quality of a part produced by high-speed machining can be increased and quality of the workpiece can be assured and tool wear while taking into account the dynamic processes occurring in the machine. The developed method for assessing the impact of the influence, takes into account the principles of forming the workpiece surface given the current technical condition of equipment and the high speed rotation of the spindle. In order to use this method it is necessary to model tool vibrations and the workpiece; analysis and modeling of the intensity of the tool's self-oscillation; modeling of tribotechnical properties of the tool carbide materials and their stability to retain their shape due to wear.

2. The Mathematical Model of Oscillations, Taking into Account Dynamic Processes Take Place in the Elastic System of Production Equipment

The assessment of the influence of vibrations of the tool and the workpiece on tool wear and surface quality was carried out by simulating vibrations of the working parts of the machine, tool wear and the shaping process itself. The model has a modular structure, where each module is designed to solve specific tasks, which include [1]: the definition of the spectrum of external perturbations; determining the dynamic characteristics of the metal-cutting machine; determining the dynamic characteristics of the cutting process, friction and tool wear; the definition of spectrum of external relative perturbations of the working parts of the metal-cutting machine, the formation of waviness and roughness of a surface.

The block diagram of the mathematical model is shown in Fig. 1.Initial input data include: metal-cutting machine's design parameters: size, shape and layout of the metalcutting machine parts; machining data: cutting parameters; dimensions of the cutting part of the tool; the strength characteristics of the material of the workpiece; the deformation characteristics of the machining layer; performance parameters of the metal-cutting machine: the machine accuracy class; possible errors of parts of the machine; assembly errors of the metal-cutting machine components.

A metal-cutting machine drives can have the following error due to manufacture and assembly: the imbalance of the spindle and drive components; inaccuracy of manufacture and assembly of rolling bearings, roller ways, rolling screwmotion drive; inaccuracy of manufacture and assembly of gears and belt transmissions; manufacturing and assembly errors of components of motors. These errors produce cyclical vibrations.

The cutting process is an element of the metal-cutting machine's dynamic system, the input parameter of the cutting process is the relative vibrations of working elements of the metal-cutting machine, the output parameter is the cutting force acting on the elastic system of the metalcutting machine. Relative oscillations of the working elements of the metal-cutting machine cause changing of the cutting force that leads to decreasing machining accuracy.

Vibrations of the tool occur during cutting are determined by many factors, including static and dynamic properties of the machine-workpiece-tool system, tool geometry, technological parameters of cutting process, physical and mechanical characteristics of materials, frictional interaction between the tool and the workpiece, and etc.

* E-mail address: mss@ugatu.su ISSN: 1791-2377 @ 2015 Kavala Institute of Technology. All rights reserved. 


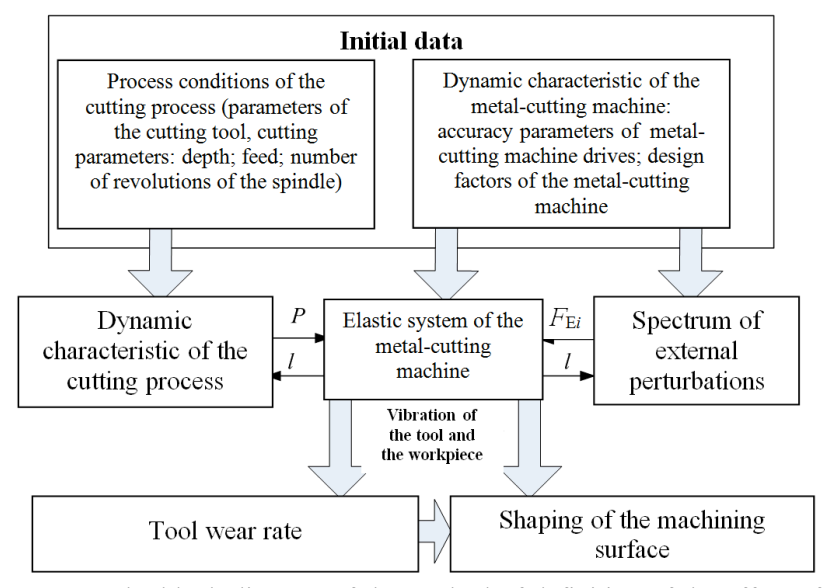

Fig. 1. The block diagram of the method of definition of the effect of vibration of working elements of the metal-cutting machine on the machined surface: $P$ - force of cutting, $F_{\mathrm{Ei}}$ - external perturbing forces, $l$ - the movement of the working elements of the metal-cutting machine.

Physical phenomena that characterize the process of chip formation, lead to a change of the cutting force with respect to the change of factors that caused it, thus giving the cutting process its inertial characteristics. The transfer function of the cutting process, taking into account the work of V.A. Kudinov [2], can be expressed as the ratio:

$$
W_{p}=\frac{P}{h}=\frac{K_{p}}{T_{p} p+1}
$$

where $P$ - the force of cutting; $h$ - the cutting depth; $T_{p}-$ time constant of chip; $K_{p}-$ the coefficient of cutting depends on the cutting force and the cutting parameters and the type of treatment.

Machining of metals with a multiple-cutting-edge tool involves the preparation of several cutting blades, where each blade cuts after the previous one [3]. In this case, the transfer function of the cutting process is determined by the expression

$$
W_{p}=\frac{P}{h}=\frac{K_{p}\left(1-e^{q p}\right)}{T_{p} p+1}
$$

where $\tau=h / Z$ - the magnitude of the delay, depending on the number of tool blades; $h$ - the cutting depth, $Z$-the amount of blades.

Taking into account the above principles, in order to create the design scheme of the dynamic model of the metalcutting machine the following assumptions were made:

- the external load is concentrated;

- the spindle, drive shafts, the frame and equipment are represented as spring beams;

- parts that are mounted on the spindle and the shaft are represented as lumped masses which are rigidly connected to the beam elements;

- $\quad$ rolling bearings have radial and axial linear stiffness characteristics;

- fixed couplings of the spindle with arbor and other elements are represented as elastic-dissipative elements with radial, angular and torsional stiffness;

- movable couplings are represented as elasticdissipative elements with unit stiffness;
- $\quad$ perturbation effects of bearings and shaft drives are transmitted to the frame through the wall of the drive case which experience longitudinal oscillations;

- drive transmissions (gears and belts) are considered as elements with radial and torsional stiffness;

- the relationship between stress and strain in the material of elements of the metal-cutting machine obey Hooke's law.

The model is based on these assumptions that allow to describe the dynamics of the elastic system (ES) of the metal-cutting machine with a system of linear second order differential equations.

The system of differential equations describing the relative oscillation of the tool and the workpiece for drives of the metal-cutting machine consists of $n$ equations, where $n$ - number of arrays. For the $i$-th array balance equation takes the form of operating forces

$m_{i} \overline{\ddot{l}}_{i}+h_{i} \overline{\dot{l}}_{i}+c_{i} \bar{l}_{i}=\sum_{i}^{n} F_{B i}$,

where $m_{i}$ - the weight of the unit; $h_{i}$-damping of the unit; $c_{i}$ - the rigidity of the unit; $l_{i}$ - the movement of the unit in the direction of one of the axes $\mathrm{X}, \mathrm{Y}$ or $\mathrm{Z}$.

The left side of each equation represents the action of the internal forces and the force of inertia in the $i$-th unit of the metal-cutting machine, and the right side of each equation represents the effect of the active external forces $F_{B}$ in the $i$-th unit.

There are several types of treatment each one has its own movements of working elements of the metal-cutting machine. This leads to the fact that the normal to the surface of the workpiece will be variable with respect to the machine coordinate system. It is necessary to consider the type of the interpolation to treat complex surfaces. In this case, the amplitude of the resulting oscillation vector of the tool and the workpiece's oscillation vector have to be calculated, taking into account movements of the metal-cutting machine's drives accordingly to the block diagram of the metal-cutting machine.

Perturbation actions in the electric motors of drives in the screw drive system with roller ways and in the main motion drive leads to vibrations of the tool with an amplitude $H_{j t}$ and frequency $\omega_{j t}$ and to vibrations of the part with an amplitude $H_{j p}$ and frequency $\omega_{j p}$. Vibrations of the working elements of the metal-cutting machine acting on an equivalent elastic system of the metal-cutting machine, change cutting parameters. Vibrations occuring on the plane parallel to the machined surface, cause changes $d S$ of feed $S$, thus the actual feed is the sum $S_{a}=S+d S$. Vibrations in the normal direction to the machining surface cause changes in the cutting depth $t$ on the magnitude of the vibration amplitude, which leads to errors in shaping due to the surface roughness and waviness of the treated surface.

In order to determine the absolute amplitudes of the vibration of the $n$-th unit of the design of the metal-cutting machine it is necessary to divide the whole range of frequencies investigated in the band width $\Delta f$. The rootmean-square amplitude of the vibration displacement of the $j$-th coordinate displacement vector in the band width $\Delta f$ is equal to: 
$A_{j}(\Delta f)=\sqrt{\sum_{s} \sum_{\Delta f} H_{k j}^{2}}$,

where $s$ - the of sources of vibration.

Knowing the amplitudes of the $k$-th harmonic of the $j$-th coordinate in the frequency band of the displacement vector due to vibrations of the working elements of the metalcutting machine, the amplitude of their relative vibrations can be found in the same frequency band. The relative displacements of the working elements of the metal-cutting machine are only considered in the plane normal to the machining surface of the workpiece.

The amplitude of vibrations can be represented as the root-mean-square sum of amplitudes of the $k$-th harmonic of the $j$-th coordinate of vectors of vibro-displacements of working elements.

$$
A_{k j, r e l}(\Delta f)=\sqrt{\sum_{s} \sum_{\Delta f}\left(H_{k j t}^{2}+H_{k j w}^{2}\right)},
$$

where $H_{k j t}^{2}$ and $H_{k j w}^{2}$ - the amplitudes of the $k$-th harmonic of $j$-th coordinate of vibro-displacement vectors of working elements of metal-cutting machine.

\section{Effect of intensity of vibrations of the tool and the workpiece on the tool life}

Performing longitudinal turning of the high-temperature alloy nickel-based alloy HN60V with the VK6M carbide tool at a cutting speed of $15-60 \mathrm{~m} / \mathrm{min}$ showed the dependence of the vibration velocity $A(f)$ on the tool vibration frequency $f$ (fig. 2). [4]

The graph of the relationship shows, there are two distinct bands on the graph: the low-frequency band in the frequency band $20-300 \mathrm{~Hz}$ and the high-frequency band - in the frequency band $2000-4000 \mathrm{~Hz}$. Within these bands the amplitude increases abruptly.

The low-frequency band is caused by vibrations of unbalanced elements of the metal-cutting machine and etc. The high-frequency band is caused by the eigenfrequency of the cutting tool. These vibrations are mostly determined by materials of the tool and the workpiece, and also by process variables of the cutting process.

Thus, the whole frequency range can be divided into three bands. The first band of vibrations for frequencies 20$150 \mathrm{~Hz}$. At this frequency range the optimal vibration amplitude is equal $30-150 \mu \mathrm{m}$. The machinability in this band is provided by chip control, as well as by supplying a coolant to the cutting blades to improve the cooling efficiency of the tool. Vibrations with this intensity facilitate the process of plastic deformation without causing damage and reducing tool life. The second band of vibrations are for frequencies $150-1000 \mathrm{~Hz}$. In this band optimal vibrations are vibrations with amplitudes of 8-20 $\mu \mathrm{m}$. Maintaining the amplitude at this level increases tool life and surface quality. This level does not cause heavy wear and destruction of the tool. The third band has a frequency of $15-35 \mathrm{kHz}$. There are optimal amplitudes of 1-5 $\mu \mathrm{m}$ in this band. These vibrations increase machinability by facilitating plastic deformation, reducing cutting forces and friction, reducing the adhesion phenomena on the areas of contact with the workpiece and chip.
The results of experimental studies of the amplitudefrequency response of the edge cutting machining showed an association between the maximum of resonance amplitudes of high-frequency tool vibrations to the minimum of the tool wear rate.

Analysis of experimental data [4] has shown that the amplitude of high-frequency vibrations essentially depends on the materials of the tool and the part as well as the cutting

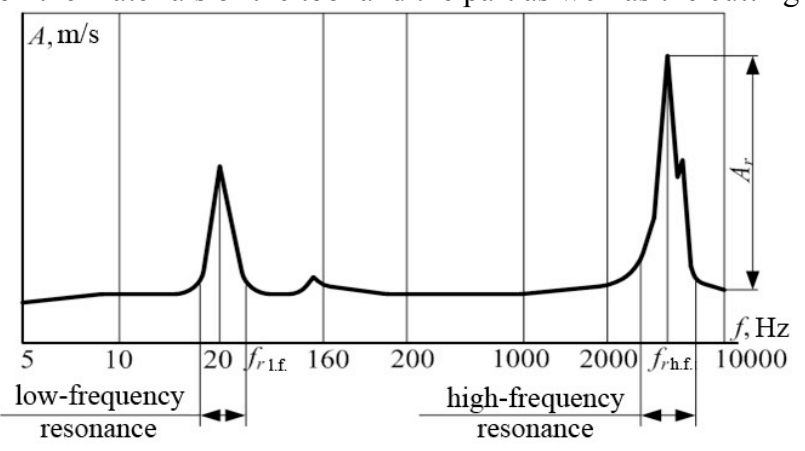

Fig. 2. The characteristic spectral dependence of the vibration velocity $A(f)$ on the tool vibration frequency $f$.

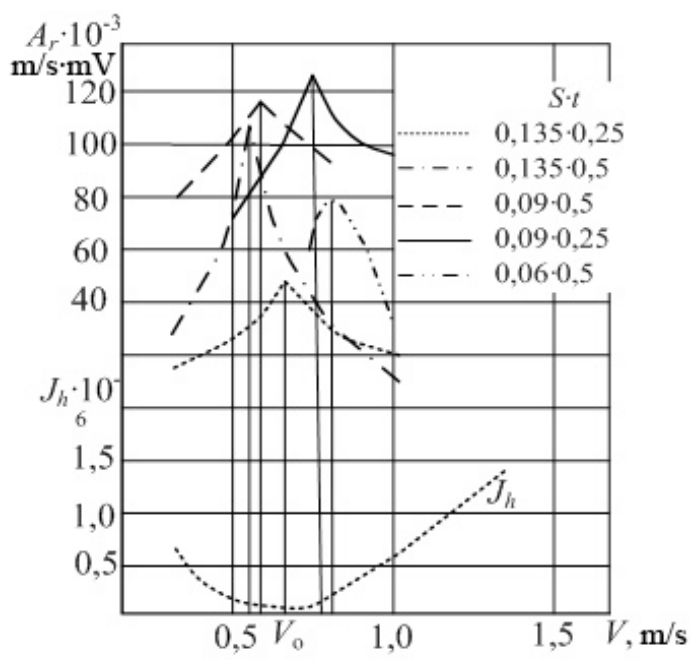

Fig. 3. Effect of high-amplitude vibrations and cutting speed on the tool wear rate (VK10HOM) during turning of the HN35VTYU-VD alloy workpiece.

parameters. It was found that the amplitude of highfrequency vibrations (fig. 3) reaches its maximum at cutting speed that corresponds to the minimum of the tool wear rate.

The experimental data confirm that the proposed mathematical model adequately describes the impact of the tool vibrations and the workpiece on the tool wear rate taking into account the dynamic processes of the metalcutting machine drives, frictional forces, cutting parameters and external vibrational perturbations.

\section{Effect of intensity of vibration of the tool, the workpiece and tool wear on shaping of the surface}

The dependence between the waviness and roughness parameters of the part and vibrations of the tool and the workpiece can be found by analyzing the forming process of the part $[5,6]$. The forming vector $r_{0}$ in this case is determined not only by vibrations of the working elements of the metal-cutting machine and the tool cutting edge 
geometry, but also by the magnitude of tool cutting edge's wear.

As a result, the practical implementation of the model in relation to the CNC machining centers, was found that the amplitude-frequency spectrum of vibrational perturbations of the metal-cutting machine drives corresponds to certain manufacturing errors of drive elements, it is an essential diagnostic character of process equipment, which allows to assess the impact of the dynamic of vibrations on the elements of the elastic system, including the tool wear rate.

\section{References}

[1]. Yu. V. Idrisova, R. G. Kudoyarov, S. I. Fetsak, "Modeling of relative vibrations of the working elements of the metal-cutting machine", Vestnik UGATU, vol. 17, no.8, p. 90-96, 2013. [in Russian]

[2]. Kudinov V. A. Dynamics of machine tools. Moscow: Mashinostroenie, 1969. [in Russian]

[3]. J. N. Sankin, Dynamics of machine tool supporting system. Moscow: Machinostroenie, 1986. [in Russian]

[4]. V. V. Postnov, B. U. Sharipov, and L. S. Shuster, Processes on the contact surfaces, tool wear and surface finish properties. Sverdlovsk: Publishing House of the Urals. University Press, 1988. [in Russian]

[5]. R. G. Kudoyarov, S. I. Fetsak, Yu. V. Idrisova "Modeling Surface Characteristics of Finished Parts", Journal of Engineering Science and Technology Review, vol.5, issue 7, pp. 20-23, 2014.

[6]. R. G. Kudoyarov, S. I. Fetsak, Yu. V. Idrisova "Research of influence of forced oscillations of the metal-cutting machine to form the workpiece surface", Improving the efficiency of the machining on the basis of modeling of physical phenomena. Materials of All-Russian Scientific and Technical Conference. Rybinsk, part.2, pp.162-166, 2009. 